\title{
Children of Senegal River Basin show the highest prevalence of Blastocystis sp. ever observed worldwide
}

Dima El Safadi ${ }^{1,2 \dagger}$, Lobna Gaayeb ${ }^{1,3+}$, Dionigia Meloni ${ }^{1,4}$, Amandine Cian ${ }^{1}$, Philippe Poirier ${ }^{5}$, Ivan Wawrzyniak ${ }^{5}$, Frédéric Delbac ${ }^{5}$, Fouad Dabboussi ${ }^{2}$, Laurence Delhaes ${ }^{1}$, Modou Seck ${ }^{3}$, Monzer Hamze ${ }^{2}$, Gilles Riveau ${ }^{1,3}$ and Eric Viscogliosi ${ }^{1 *}$

\begin{abstract}
Background: Blastocystis sp. is currently the most common intestinal protist found in human feces and considered an emerging parasite with a worldwide distribution. Because of its potential impact in public health, we reinforced the picture of Blastocystis sp. prevalence and molecular subtype distribution in Africa by performing the first survey of this parasite in Senegal.

Methods: Stool samples from 93 symptomatic presenting with various gastrointestinal disorders or asymptomatic children living in three villages of the Senegal River Basin were tested for the presence of Blastocystis sp. by nonquantitative and quantitative PCR using primer pairs targeting the SSU rDNA gene. Positive samples were subtyped to investigate the frequency of Blastocystis sp. subtypes in our cohort and the distribution of subtypes in the symptomatic and asymptomatic groups of children.

Results: By the use of molecular tools, all 93 samples were found to be positive for Blastocystis sp. indicating a striking parasite prevalence of $100 \%$. Mixed infections by two or three subtypes were identified in eight individuals. Among a total of 103 subtyped isolates, subtype 3 was most abundant (49.5\%) followed by subtype 1 (28.2\%), subtype 2 (20.4\%) and subtype 4 (1.9\%). Subtype 3 was dominant in the symptomatic group while subtypes 1 and 2 were detected with equal frequency in both symptomatic and asymptomatic groups. The distribution of subtypes was compared with those available in other African countries and worldwide. Comparison confirmed that subtype 4 is much less frequently detected or absent in Africa while it is commonly found in Europe. Potential sources of Blastocystis sp. infection including human-to-human, zoonotic, and waterborne transmissions were also discussed.

Conclusions: The prevalence of Blastocystis sp. in our Senegalese population was the highest prevalence ever recovered worldwide for this parasite by reaching 100\%. All cases were caused by subtypes 1, 2, 3 and 4 with a predominance of subtype 3. More than half of the children infected by Blastocystis sp. presented various gastrointestinal disorders. Such high prevalence of blastocystosis in developing countries makes its control a real challenge for public health authorities.
\end{abstract}

Keywords: Blastocystis sp, Intestinal parasite, Molecular epidemiology, Pathogenicity, PCR, Subtyping, Transmission, Zoonosis

\footnotetext{
*Correspondence: eric.viscogliosi@pasteur-lille.fr

${ }^{\dagger}$ Equal contributors

'Institut Pasteur de Lille, Centre d'Infection et d'Immunité de Lille (CIIL), Inserm U1019, CNRS UMR 8204, Université Lille Nord de France, 1 rue du Professeur Calmette, BP 245, 59019 Lille cedex, France

Full list of author information is available at the end of the article
} 


\section{Background}

Blastocystis sp. is an anaerobic protist that inhabits the gastrointestinal tract of humans and many groups of animals [1-4]. This cosmopolitan enteric parasite with a worldwide distribution [5] is often identified as the most common unicellular eukaryote reported in human fecal samples [1]. Indeed, its prevalence may exceed $50 \%$ in developing countries [6,7] and reach $20 \%$ in industrialized countries $[8,9]$. These differences may be explained by poor sanitary conditions, close animal contact, and consumption of contaminated food or water [10-12]. Such prevalence also suggests large-scale human-tohuman [13], waterborne, and zoonotic transmissions for Blastocystis sp. [14]. Numerous studies have demonstrated the resistance of cysts of the parasite in feces and environmental sources [15] highlighting the fecal-oral route as the main mode of transmission of Blastocystis sp. Moreover, a higher risk of infection has been identified in food and animal handlers, providing conclusive evidence on the transmission of the parasite between humans and animals $[16,17]$.

At the morphological level, Blastocystis sp. isolates from humans and animals have been reported to be roughly indistinguishable [1]. However, extensive genetic diversity among Blastocystis sp. isolates has been reported based on the comparison of the small-subunit (SSU) rDNA gene sequence [18] leading to the classification of Blastocystis sp. isolates into subtypes (STs) [19]. To date, according to recent epidemiological surveys in human and animal populations, 17 STs have been identified [2] but it is highly likely that other STs remain yet to be uncovered. Among the 17 STs, nine of them have been detected in humans with a varying prevalence [5]. Indeed, only four of them are frequent (ST1, ST2, ST3 and ST4) and represent around 90\% of the subtyped isolates. A majority of human infections with Blastocystis sp. is attributable to ST3 but the distribution of the four predominant STs may vary among the areas studied. Thereby, ST4 is almost as common as ST1 and ST3 in some European countries but seems rare or completely absent in Africa, Asia, and America $[5,20]$. The other STs that are common in animal hosts i.e. ST5 to ST9, are rarely found in humans and are most likely the result of zoonotic transmission.

As Blastocystis sp. can be found in both symptomatic and asymptomatic patients its clinical relevance remained uncertain [1]. However, a converging bundle of recent in vivo, in vitro and genomic data strongly suggests that this microorganism may be pathogen [21-24] and allows proposing a model for pathogenesis of this parasite [25] particularly involving virulence factors as cysteine proteases $[26,27]$. Therefore, Blastocystis sp. should be associated with a variety of non-specific gastrointestinal disorders including diarrhea, abdominal pain, nausea and vomiting $[1,22]$ and also suspected to be linked to irritable bowel syndrome (IBS) $[25,28]$ and chronic or acute urticarial lesions [29,30]. In addition, Blastocystis sp. has increasingly been implicated in diarrheal illness in immunocompromised individuals including HIV/AIDS and cancer patients and transplant recipients [31,32].

It has long been suggested that the pathogenesis of Blastocystis sp. may be dependent upon ST but recent epidemiological data remain contradictory [8,29,33-36]. Nevertheless, the likely pathogenic power of Blastocystis sp. coupled to its high prevalence in the human population raise crucial questions about its current burden in public health. Consequently, information on the prevalence of the parasite and the distribution of STs are starting to emerge in still poorly studied geographic areas as Africa. To date the available data concern only few African countries of the North and West such as Egypt [21,37,38], Libya, Nigeria, and Liberia [5] and one Eastern African country, Tanzania [39]. Therefore, the aim of the present study was to reinforce the picture of Blastocystis sp. prevalence and ST distribution in Africa by performing the first survey of this parasite in Senegal. In this epidemiological study, we described the prevalence of Blastocystis sp. infection and ST distribution in a cohort of Senegalese children living in 3 rural villages in close contact with animals and with poor or no access to clean water and sanitation. The distribution of Blastocystis sp. STs in both asymptomatic and symptomatic individuals was investigated together with potential risk factors for transmission of the parasite.

\section{Methods}

\section{Cohort and collection of samples}

This work has been carried out as part of the "SchistoVAN" project (clinicaltrials.gov ID NCT01553552), sponsored by the Biomedical Research Centre EPLS (Saint-Louis, Senegal) (www.espoir-sante.org). The present study was approved by the National Ethics Committee of the Ministry of Health of Senegal (September 2011; protocol number SEN11/43, clinicaltrials.gov: NCT01553552). Oral and written informed consents were obtained from the parents or the legal guardians of the children in accordance with the Code of Ethics of the World Medical association (Declaration of Helsinki). Children enrolled in the present study formed a sub-cohort of the "AnoPalAnoVac" project (clinicaltrials.gov ID NCT01545115) and were recruited on the basis of their age (6 to 10 years of age in October 2011) through village nurses, healthcare workers in the community and school directors. Date of birth was ascertained from vaccination cards or school register. A standardized questionnaire was completed for each child (see Additional file 1). In particular, children were asked about each gastrointestinal symptom individually including abdominal pain, diarrhea, and vomiting. 
This cross-sectional study was conducted in October 2011, in 3 villages of the Podor district located in northern Senegal: Agniam Towguel $\left(16^{\circ} 32^{\prime} \mathrm{N}-14^{\circ} 48^{\prime} \mathrm{W}\right.$; total population (TP): 989; several temporary ponds, traditional housing, irrigated crops), Fanaye Diery $\left(16^{\circ} 32^{\prime} \mathrm{N}-15^{\circ} 13^{\prime} \mathrm{W}\right.$; TP: 6781; animal husbandry, irrigated crops, some urbanized habitat), and Niandane ( $16^{\circ} 35^{\prime} \mathrm{N}-14^{\circ} 59^{\prime} \mathrm{W}$; TP: 5100 ; rice and banana farming, irrigated crops, some urbanized habitat, no access to running water). In this region, the climate is Sahelian, with annual rainfall between July and September. Mean temperature ranges between $20^{\circ} \mathrm{C}$ and $30^{\circ} \mathrm{C}$ during the cool season (November to February) and between $25^{\circ} \mathrm{C}$ and $38^{\circ} \mathrm{C}$ during the warm season (March to October). In this study, stool samples were collected from 93 symptomatic and asymptomatic children $($ Agniam $=17$; Fanaye $=38$; Niandane $=38)$ presenting or not gastrointestinal disorders. These boys $(n=46)$ and girls $(n=47)$ were aged 6 to 10 years (median age 8.4; quartiles: 6.6; 9.5) (Table 1). Children presented no symptoms of severe illness and/or fever and were subject to a questionnaire that was designed to collect clinical data especially regarding recent diarrhea, vomiting, and abdominal pain. For storage and transport at $-20^{\circ} \mathrm{C}$ to Lille, stool samples were added in Stool Transport and Recovery (S.T.A.R.) buffer (Roche) in a ratio of 1:3 according to the manufacturer's recommendations then homogenized by shaking.

\section{DNA extraction and molecular subtyping of Blastocystis sp. isolates}

Samples stored in S.T.A.R. buffer were stirred and then centrifuged for $1 \mathrm{~min}$ at 1,000 g. Total genomic DNA was extracted from $200 \mu \mathrm{l}$ of the cleared stool supernatant using the QiaAMP DNA Stool Mini Kit (Qiagen, Hilden, Germany) according to the manufacturer's instructions. For each sample, $5 \mu \mathrm{l}$ of extracted DNA was submitted to non-quantitative Polymerase Chain Reactions (non-qPCRs) as previously described using two independent pairs of Blastocystis sp.-specific primers designed by Scicluna et al. [40] and Poirier et al. [20], both targeting the small subunit (SSU) rDNA coding region. The respective $600 \mathrm{bp}$ - and $320 \mathrm{bp}$-amplified domains have been shown to contain sufficient information for accurate subtyping of Blastocystis sp. isolates [20,40]. Non-qPCR amplifications were carried out in $50 \mu \mathrm{l}$ according to standard conditions for Platinum Taq HighFidelity DNA polymerase (Invitrogen). For each DNA sample, the non-qPCR product with the highest intensity on agarose gel was purified and sequenced. Direct sequencing of few non-qPCR products generated mixed signals that could reflect infections by different STs or was of poor quality probably due to a too small amount of parasite DNA matrix. Because of the difficulty in interpreting the corresponding DNA sequencing chromatograms, same samples were re-analyzed by non-qPCRs and positive bands of the expected sizes were purified and cloned as previously described [41]. Three positive clones containing inserts were arbitrarily selected for each sample and sequenced on both strands. The DNA samples negative by non-qPCRs were subsequently amplified using the real time qPCR assay developed by Poirier et al. [20]. Due to the high sensitivity of this detection method, various controls were performed to prevent artifacts related to contamination through different sources: DNA extraction controls (isolation of DNAs without stool and from a Blastocystis sp.-negative stool) subsequently used in qPCR assays and negative (DNA matrix replaced by water) and positive (DNA obtained from a Blastocystis sp. ST4 culture) qPCR controls. The expected $320 \mathrm{bp}$-amplified variable region of the SSU rRNA gene was directly sequenced for subtyping. The SSU rRNA gene sequences obtained in this study have been deposited in GenBank under accession numbers KF848509 to KF848624. Obtained sequences were compared with all Blastocystis sp. SSU rRNA gene sequences available from the National Centre for Biotechnology Information (NCBI) using the BLAST program. STs were identified by determining the exact match or closest similarity against all known Blastocystis sp. STs according to the updated classification by Alfellani et al. [2].

\section{Results and discussion}

Screening for Blastocystis sp. in a cohort of Senegalese children using molecular methods

In this study, fecal samples were collected from 93 children, 46 boys and 47 girls, aged 6 to 10 years and living in 3 villages situated in the north of Senegal. This cohort consisted of two groups: one symptomatic composed of 54 children presenting variously with abdominal pain, vomiting, and diarrhea and one asymptomatic including 39 other children (Table 1). All 93 samples were screened for Blastocystis sp. by non-qPCR and qPCR methods using specific primer pairs amplifying distinct domains of the SSU rDNA coding region $[20,40]$. NonqPCR products were directly sequenced or subsequently cloned whether the resulting sequence chromatogram showed double or poor quality signals. In the latter case that involved 32 samples, three positive clones containing inserts were arbitrarily selected and sequenced. Overall, 71 samples were identified as positive for Blastocystis sp. by non-qPCR methods. The remaining 22 samples negative by non-qPCR nevertheless proved to be positive for the parasite by qPCR. Thereby, by means of these different molecular approaches, Blastocystis sp. was recognized in $100 \%$ of stool samples of this Senegalese population. To our knowledge this represents the highest prevalence ever recovered worldwide for this parasite. 
Table 1 Clinical data and Blastocystis sp. STs among symptomatic and asymptomatic patients in Senegal

\begin{tabular}{|c|c|c|c|c|c|c|}
\hline Patients & Sex/age & Symptoms & $\begin{array}{l}\text { Blastocystis sp. ST } \\
\text { by non-qPCR }\end{array}$ & $\begin{array}{l}\text { Nucleotide } \\
\text { differences }{ }^{b}\end{array}$ & $\begin{array}{l}\text { Blastocystis sp. } \\
\text { ST by qPCR }\end{array}$ & Accession no. \\
\hline DSS1 & $M / 6.2$ & Abdominal pain & & & 1 & KF848509 \\
\hline DSS2 & $F / 5.9$ & Abdominal pain, diarrhea & $3(S c)$ & 0 & & KF848510 \\
\hline DSS3 & $M / 6.8$ & & $3(S c)$ & & & KF848511 \\
\hline DSS4 & $F / 6.1$ & Abdominal pain, vomiting & $1(\mathrm{Sc})$ & & & KF848512 \\
\hline DSS5 & $M / 8.3$ & & & & 3 & KF848513 \\
\hline DSS6 & $M / 7.3$ & Abdominal pain, diarrhea & 1 (Sc) & & & KF848514 \\
\hline DSS7 & $F / 5.9$ & & & & 3 & KF848515 \\
\hline DSS8 & $M / 6.8$ & Abdominal pain, vomiting & $1(\mathrm{Sc})$ & & & KF848516 \\
\hline DSS9 & $F / 9.2$ & Abdominal pain & & & 3 & KF848517 \\
\hline DSS10 & M/9.7 & Vomiting & $3(S c)$ & 0 & & KF848518 \\
\hline DSS11 & $F / 10$ & Diarrhea, vomiting & $3(S c)$ & & & KF848519 \\
\hline DSS12 & M/8.8 & Abdominal pain, vomiting & & & 3 & KF848520 \\
\hline DSS13 & $F / 8.6$ & Abdominal pain & $1(\mathrm{Sc})$ & & & KF848521 \\
\hline DSS14 & $\mathrm{M} / 7$ & & $2(\mathrm{Sc})$ & 0 & & KF848522 \\
\hline DSS15 & $F / 7.7$ & & $2(S c)$ & & & KF848523 \\
\hline DSS16 & $M / 6.9$ & & & & 3 & KF848524 \\
\hline DSS17 & $F / 7.7$ & & & & 3 & KF848525 \\
\hline DSS18 & $F / 6.1$ & & $3(\mathrm{Sc})$ & 0 to14 & & KF848526-7 \\
\hline DSS19 & $M / 8.7$ & & & & 2 & KF848528 \\
\hline DSS20 & M/9.7 & Abdominal pain & $2(\mathrm{Sc})$ & & & KF848529 \\
\hline DSS21 & $F / 6$ & Abdominal pain & 1 (Sc) & & & KF848530 \\
\hline DSS22 & $M / 10.1$ & Abdominal pain & $3(S c)$ & & & KF848531 \\
\hline DSS23 & $\mathrm{M} / 7.7$ & & Mixed 2 and $3(P)$ & 0 & & KF848532-3 \\
\hline DSS24 & $F / 6.4$ & & $1(\mathrm{Sc})$ & & & KF848534 \\
\hline DSS25 & $F / 9.6$ & Abdominal pain & Mixed 3 and 4 (Sc) & 0 to 4 & & KF848535-7 \\
\hline DSS26 & $F / 6.5$ & Abdominal pain & Mixed 1, 2 and $3(\mathrm{Sc})$ & & & KF848538-40 \\
\hline DSS27 & $M / 7.2$ & & $3(\mathrm{Sc})$ & & & KF848541 \\
\hline DSS28 & $\mathrm{M} / 8$ & & $3(S c)$ & & & KF848542 \\
\hline DSS29 & $F / 7.3$ & & & & 2 & KF848543 \\
\hline DSS30 & $M / 5.8$ & Abdominal pain & & & 1 & KF848544 \\
\hline DSS31 & $F / 8.6$ & Abdominal pain & $2(\mathrm{Sc})$ & & & KF848545 \\
\hline DSS32 & $M / 6.6$ & & $3(S c)$ & & & KF848546 \\
\hline DSS33 & $\mathrm{M} / 7.7$ & Abdominal pain & Mixed 2 and 3 (Sc) & 0 & & KF848547-8 \\
\hline DSS34 & M/9.5 & Vomiting & $3(\mathrm{Sc})$ & & & KF848549 \\
\hline DSS35 & $M / 7.7$ & Abdominal pain & $3(S c)$ & 0 & & KF848550 \\
\hline DSS36 & $F / 5.9$ & Abdominal pain & $3(\mathrm{Sc})$ & & & KF848551 \\
\hline DSS37 & $M / 7.4$ & & $2(S c)$ & 0 & & KF848552 \\
\hline DSS38 & $F / 9.7$ & Abdominal pain, diarrhea, vomiting & 1 (Sc) & 0 & & KF848553 \\
\hline DSS39 & $F / 9.8$ & Abdominal pain & & & 3 & KF848554 \\
\hline DSS40 & $F / 9.9$ & Abdominal pain, diarrhea, vomiting & $2(\mathrm{Sc})$ & & & KF848555 \\
\hline DSS41 & $F / 9.7$ & Vomiting & $3(S c)$ & 0 & & KF848556 \\
\hline DSS42 & $F / 10.2$ & Abdominal pain, diarrhea & $1(\mathrm{Sc})$ & & & KF848557 \\
\hline DSS43 & M/9.3 & & $2(S c)$ & & & KF848558 \\
\hline
\end{tabular}


Table 1 Clinical data and Blastocystis sp. STs among symptomatic and asymptomatic patients in Senegal (Continued)

\begin{tabular}{|c|c|c|c|c|c|c|}
\hline DSS44 & $M / 10$ & Abdominal pain & $3(S c)$ & & & KF848559 \\
\hline DSS45 & $F / 8.9$ & Abdominal pain & $3(S c)$ & 0 to 14 & & KF848560-1 \\
\hline DSS46 & $F / 10.2$ & Abdominal pain & & & 3 & KF848562 \\
\hline DSS47 & $F / 10$ & & $3(\mathrm{Sc})$ & 0 & & KF848563 \\
\hline DSS48 & M/9.8 & & $3(S c)$ & 0 & & KF848564 \\
\hline DSS49 & $F / 10$ & & $2(\mathrm{Sc})$ & & & KF848565 \\
\hline DSS50 & $F / 10.2$ & & & & 1 & KF848566 \\
\hline DSS51 & $M / 9.4$ & & $1(\mathrm{Sc})$ & & & KF848567 \\
\hline DSS52 & $M / 9$ & Abdominal pain & $1(\mathrm{Sc})$ & & & KF848568 \\
\hline DSS53 & $F / 8.1$ & & $1(\mathrm{Sc})$ & & & KF848569 \\
\hline DSS54 & M/8.5 & & Mixed 1 and 2 (Sc) & 0 & & KF848570-1 \\
\hline DSS55 & M/9.1 & Abdominal pain & $3(S c)$ & & & KF848572 \\
\hline DSS56 & $F / 8.6$ & & $1(\mathrm{Sc})$ & & & KF848573 \\
\hline DSS57 & $F / 9.7$ & Abdominal pain & $2(\mathrm{Sc})$ & & & KF848574 \\
\hline DSS58 & M/9.3 & Abdominal pain & $2(\mathrm{Sc})$ & & & KF848575 \\
\hline DSS59 & M/8.9 & Abdominal pain & $3(S c)$ & & & KF848576 \\
\hline DSS60 & $F / 8.8$ & & $3(\mathrm{Sc})$ & & & KF848577 \\
\hline DSS61 & M/9.9 & & & & 1 & KF848578 \\
\hline DSS62 & $F / 9.5$ & Abdominal pain & & & 1 & KF848579 \\
\hline DSS63 & $M / 8.5$ & Abdominal pain & $1(\mathrm{SC})$ & & & KF848580 \\
\hline DSS64 & $M / 6.2$ & Abdominal pain & $3(\mathrm{Sc})$ & & & KF848581 \\
\hline DSS65 & M/9.8 & & $1(\mathrm{Sc})$ & & & KF848582 \\
\hline DSS66 & $F / 9.9$ & Abdominal pain & $2(\mathrm{Sc})$ & & & KF848583 \\
\hline DSS67 & $F / 6.1$ & Abdominal pain & Mixed 1 and 4 (Sc) & 0 & & KF848584-5 \\
\hline DSS68 & $F / 6.1$ & & $1(\mathrm{Sc})$ & 0 & & KF848586 \\
\hline DSS69 & $F / 6.8$ & & & & 1 & KF848587 \\
\hline DSS70 & $F / 7.8$ & Abdominal pain & $3(\mathrm{Sc})$ & & & KF848588 \\
\hline DSS71 & $F / 6.7$ & Abdominal pain & & & 3 & KF848589 \\
\hline DSS72 & $M / 6$ & Abdominal pain, vomiting & $3(\mathrm{Sc})$ & & & KF848590 \\
\hline DSS73 & $F / 7$ & Abdominal pain & $3(S c)$ & 0 & & KF848591 \\
\hline DSS74 & $M / 8.5$ & & & & 3 & KF848592 \\
\hline DSS75 & M/5.9 & Abdominal pain & & & 3 & KF848593 \\
\hline DSS76 & M/8.1 & Abdominal pain & & & 1 & KF848594 \\
\hline DSS77 & M/6.6 & Abdominal pain & $3(\mathrm{Sc})$ & 2 to 12 & & KF848595-7 \\
\hline DSS78 & $M / 6.9$ & Abdominal pain & & & 2 & KF848598 \\
\hline DSS79 & $M / 8.4$ & & $3(\mathrm{Sc})$ & 0 to 11 & & KF848599-600 \\
\hline DSS80 & $M / 6.1$ & & & & 3 & KF848601 \\
\hline DSS81 & $F / 5.9$ & Abdominal pain & Mixed 2 and 3 (Sc) & 0 & & KF848602-3 \\
\hline DSS82 & $F / 9.8$ & Abdominal pain & $3(\mathrm{Sc})$ & 0 & & KF848604 \\
\hline DSS83 & $M / 6.4$ & & & & 1 & KF848605 \\
\hline DSS84 & $F / 9.2$ & & $2(\mathrm{Sc})$ & & & KF848606 \\
\hline DSS85 & $F / 6.8$ & Abdominal pain, vomiting & $3(P)$ & 3 to 5 & & KF848607-9 \\
\hline DSS86 & $F / 6.6$ & Abdominal pain & Mixed 1, 2 and $3(P)$ & & & KF848610-2 \\
\hline DSS87 & M/9.9 & Abdominal pain & $3(P)$ & 0 & & KF848613 \\
\hline DSS88 & M/9.5 & & $1(\mathrm{P})$ & 0 to 1 & & KF848614-5 \\
\hline
\end{tabular}


Table 1 Clinical data and Blastocystis sp. STs among symptomatic and asymptomatic patients in Senegal (Continued)

\begin{tabular}{|c|c|c|c|c|c|}
\hline DSS89 & $F / 9.1$ & Diarrhea & $3(P)$ & 0 & KF848616 \\
\hline DSS90 & F/9.5 & & $3(P)$ & 0 to 1 & KF848617-8 \\
\hline DSS91 & $\mathrm{F} / 9.4$ & & $1(\mathrm{P})$ & 0 to 3 & KF848619-20 \\
\hline DSS92 & $F / 6.1$ & Abdominal pain, diarrhea & $3(P)$ & 0 to 3 & KF848621-2 \\
\hline DSS93 & $M / 6$ & & $3(P)$ & 0 to 1 & KF848623-4 \\
\hline
\end{tabular}

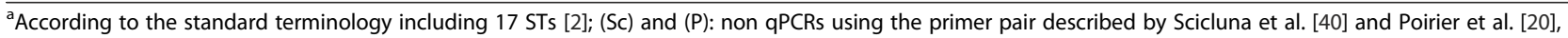
respectively.

${ }^{\mathrm{b}}$ Determined in the common region of clones belonging to the same ST and sequenced for each sample.

In almost all molecular epidemiological studies conducted to date, only one nucleic acid-based diagnostics methodology was used to identify and subtype Blastocystis sp. isolates in stool samples. Briefly, PCR screening of fecal samples can be achieved using ST-specific sequencetagged-site (STS) primers or genus-specific primers with subsequent sequencing for ST identification. Several regions in the SSU rDNA gene have been proposed by different authors for sequencing [24] but the "barcode" region designed by Scicluna et al. [40] has been extensively used as in the present study. Advantages and limitations of these two PCR approaches were recently largely discussed $[24,42]$ and a comparative study using both techniques recommended barcoding as the relevant method for Blastocystis sp. diagnosis and subtyping [43]. However, as confirmed from our data, direct sequencing of nonqPCR products may be unsuccessful in the case of mixed infections with more than one Blastocystis sp. ST in the same sample. Therefore subtyping of isolates in mixed infections requires cloning of the non-qPCR product and sequencing of several clones as previously suggested [44]. In addition, about $24 \%$ of the samples have not been diagnosed as positive for Blastocystis sp. by non-qPCR methods in our study. Hence, at this stage of our screening, the prevalence of the parasite was $76 \%$, which greatly underestimated its true prevalence as determined in a second step by qPCR (100\%). Consequently, our study confirmed the impact of detection methods to ascertain the actual prevalence of Blastocystis sp. in human populations and the interest and higher sensitivity of qPCR assays in comparison to non-qPCR methods for epidemiological surveys.

Although the prevalence of Blastocystis sp. never reached $100 \%$ in any cohort studied so far, it was described as very high in some developing countries specially, in children. By using non-molecular standard methods, the prevalence of the parasite already exceeded $40 \%$ in communities in Venezuela [45], Peru [46], Colombia [7], and in school children or orphanage in Indonesia [6], Thailand [47], and Lebanon (El Safadi unpubl.). Moreover, the prevalence of Blastocystis sp. was around $60 \%$ in immigrants in the city of Naples (southern Italy) who came for a large majority from northern and western African countries [48]. In Libya, the prevalence of the parasite has been previously reported close to $30 \%$ [49]. In Senegal, although the prevalence of Blastocystis sp. had never been determined before, intestinal parasites (protozoa and helminths) represent for a long time a major public health problem. Indeed, the global prevalence of intestinal parasites in children ranged from $32 \%$ to $84 \%$ depending on the study areas [50-53]. Such impact of intestinal parasites previously reported in the Senegalese population is clearly in accordance with the pattern found for Blastocystis sp. in the current study.

\section{Distribution of Blastocystis sp. STs in the Senegalese} population and comparison with other African countries In all the children, the expected SSU rDNA domains targeted by non-qPCR and qPCR methods were amplified and successfully sequenced. Each of the new SSU rDNA gene sequences obtained in this study showed very high similarity ( $98 \%$ to $100 \%$ ) to homologous sequences of the other Blastocystis sp. isolates reported so far. The comparison with representative sequences of all known Blastocystis sp. STs allowed the direct subtyping of the new isolates (Table 1). As stated above, for 32 of the 93 samples, cloning of the non-qPCR product was necessary for subtyping and 3 positive clones were arbitrarily selected and sequenced. For 14 of these 32 samples, the three sequenced clones were identical (Table 1). For 10 other samples presenting infection by a single ST, clones showed 1 to 14 nucleotide differences between them. As previously suggested $[40,41]$, these nucleotide differences may reflect either coinfection with two isolates of the same ST or sequence variations between SSU rDNA gene copies within the same isolate [23]. Interestingly, the higher number of nucleotide difference between the clones (11 to 14 ) was observed in children infected by ST3. Recently, by analyzing and comparing respectively SSU rDNA sequences or data from multilocus sequence typing, Meloni et al. [44] and Stensvold et al. [54] revealed substantial intra-ST diversity in ST3 and more limited intra-ST2 and intra-ST4 variations. In either 
case, we have considered in the following analysis of our data that two different clones of the same ST identified in the same patient derived from the same isolate of Blastocystis sp. For the last 8 samples, mixed infections with two (6 samples) or three STs (2 samples) were identified (Table 2). The majority of the subtyped samples included in the present study (85/93) represented single infections. With the addition of 8 mixed infections consisting of two or three different isolates, we analyzed a total of 103 isolates. As summarized in Table 3, ST3 was the most common Blastocystis sp. ST in our Senegalese population (49.5\%) followed by ST1 (28.2\%), ST2 (20.4\%) and ST4 (1.9\%). The distribution of STs was almost similar between the three villages with a majority of infections attributable to ST3. The only significant difference was that ST1 followed at position 2 in Niandiane and Agniam Towguel and at position 3 in Fanaye Diery.

The distribution of Blastocystis sp. STs in Senegal is quite similar to that observed in a majority of countries all over the world with a predominance of ST3 followed by ST1 then ST2 or ST4 [5]. If we focused on the studies previously conducted in Africa, the only available reports concerned the ST distribution in Egypt [21,37,38], Libya, Nigeria, Liberia [2], and Tanzania [39], the latter survey only including 6 isolates (Figure 1). Globally, ST1, ST2, and ST3 represent around 82\% of all 369 African isolates subtyped so far including those of the present study. ST3 is highly prevalent in African countries with an average of $46 \%$ of the 369 isolates. Consequently, because ST3 is rarely found in animals, a large-scale human-to-human transmission might likely explain the predominance of this ST in African countries as also in the rest of the world. Three studies conducted in different cities in Egypt and that in Liberia have identified ST3 as the predominant ST, as was true for our study population. However ST1 was detected with the highest frequency in Libya and Nigeria, and ST2 in Tanzania. Since ST1 and ST2 are frequently found in various animal groups including livestock $[2,4,55]$, it can be hypothesized that a not yet evaluated proportion of human infections by both STs are of zoonotic origin. Interestingly ST4 was not detected in the studies reported in Egypt, Libya and Tanzania and its prevalence is quite low in Senegal (1.9\%). Although ST4 was relatively frequent in Liberia (12\%) and Nigeria (14\%), these data confirmed that ST4 is much less frequently detected or absent in Africa while it is commonly found in Europe $[5,34]$. However, the reasons for the heterogeneous geographical distribution of ST4 remain unknown and its reservoir hosts have to be clarified. Additional STs including ST6 and ST7 were commonly found only in two studies carried out in Egypt [21,38]. Indeed, these STs were not detected in Senegal, Liberia, Nigeria, and Tanzania as in a third survey in Egypt [37] and only one isolate belonging to ST7 was identified in Libya. Both STs are usually hosted by birds (avian STs) [56] and rarely found in mammals. This was confirmed in the present study. Given their apparent host specificity, it is highly likely that human infections by avian STs are of zoonotic origin.

The prevalence of mixed ST infections was $8.6 \%(8 / 93$ samples) in our Senegalese cohort. This percentage is probably underestimated but roughly similar to that calculated (6\%) by taking into account subtyped isolates from all epidemiological studies published to date (191 of 3171 samples) and listed by Alfellani et al. [5]. Mixed ST infections were found in all three villages of our study but with different proportions. In Agniam Towguel, only one mixed infection by 3 STs was found (ST1/ST2/ ST3) (5.9\%) while 4 mixed infections by 2 or 3 STs (10.5\%) (ST2/ST3, ST3/ST4 and ST1/ST2/ST3) were recorded in Fanaye Diery. Regarding Niandane, 3 mixed infections by 2 STs (7.9\%) were identified (ST1/ST2, ST1/ ST4, and ST2/ST3) (Table 2). Mixed infections mostly corresponded to ST1, ST2, and ST3 isolates and were more likely to occur considering the high prevalence of each of these STs in our cohort. Curiously, the only two ST4 isolates of our study were identified in the context of mixed infections and not as part of single infections.

\section{Distribution of Blastocystis sp. STs in symptomatic and asymptomatic groups}

According to recent epidemiological reports, links between ST and pathogenicity are still matter of debate $[8,29,33-36,41]$. To highlight a possible relationship between ST and major intestinal symptoms associated with Blastocystis sp. infection (diarrhea, vomiting, and abdominal pain), the ST distribution was evaluated in our cohort

Table 2 Single and mixed ST infections with Blastocystis sp. identified in the Senegalese cohort

\begin{tabular}{|c|c|c|c|c|c|c|c|c|c|}
\hline \multirow[t]{2}{*}{ Villages } & \multicolumn{8}{|c|}{ Blastocystis sp. STs } & \multirow[t]{2}{*}{ Total no } \\
\hline & ST1 & ST2 & ST3 & $\mathrm{ST} 1 / \mathrm{ST} 2$ & ST1/ST4 & ST2/ST3 & ST3/ST4 & $\mathrm{ST} 1 / \mathrm{ST} 2 / \mathrm{ST} 3$ & \\
\hline Agniam Towguel & 5 & 2 & 9 & 0 & 0 & 0 & 0 & 1 & 17 \\
\hline Niandane & 13 & 5 & 17 & 1 & 1 & 1 & 0 & 0 & 38 \\
\hline Fanaye Diery & 7 & 8 & 19 & 0 & 0 & 2 & 1 & 1 & 38 \\
\hline Total no. & 25 & 15 & 45 & 1 & 1 & 3 & 1 & 2 & 93 \\
\hline
\end{tabular}


Table 3 Blastocystis sp. ST distribution among Senegalese villages

\begin{tabular}{cccccc}
\hline Villages & \multicolumn{3}{c}{ Blastocystis sp. STs (\%) } & Total no. \\
\cline { 2 - 5 } & ST1 & ST2 & ST3 & ST4 & \\
\hline Agniam Towguel & 6 & 3 & 10 & 0 & 19 \\
Niandane & 15 & 7 & 18 & 1 & 41 \\
Fanaye Diery & 8 & 11 & 23 & 1 & 43 \\
Total no. (\%) & $29(28.1)$ & $21(20.3)$ & $51(49.5)$ & $2(1.9)$ & 103 \\
\hline
\end{tabular}

of 93 symptomatic or asymptomatic children. Interestingly, the majority of children infected by Blastocystis sp. (54/93 or $58 \%$ ) presented various gastrointestinal disorders as previously observed in a pediatric unit in Turkey [57]. In the following analysis, symptomatic (6) and asymptomatic (2) children presenting mixed infections by two or three STs were removed. Thereby the remaining 48 isolates from symptomatic children and 37 isolates from asymptomatic children were considered (Table 4).
The most dominant ST in both groups was ST3, and 63\% of the ST3 isolates were found in symptomatic individuals. Predominance or high prevalence of ST3 in symptomatic groups of patients was also shown for instance in France [41], Australia [8], Turkey [57,58], and Iran [35]. Regarding ST1 and ST2 isolates, both STs were detected with equal frequency in the symptomatic and asymptomatic groups of our cohort (Chi square test, $\mathrm{p}=0.845$ ). However, ST1 was over-represented in groups of symptomatic individuals in previous epidemiological studies in China [59], Turkey [60], Iran [35], Egypt [21], and Lebanon [36]. Moreover, ST1 was the most prevalent ST in some cohorts presenting with IBS $[38,61]$ and was shown to be pathogenic in rat models of blastocystosis [21]. In contrast, ST2 was not usually overrepresented in groups of symptomatic patients from different countries and Dogruman-Al et al. [57] suggested that ST2 was a nonpathogenic ST. However, in our study, the proportion of ST2 isolates in symptomatic patients was far from negligible. Moreover, all ST2 isolates identified in French and

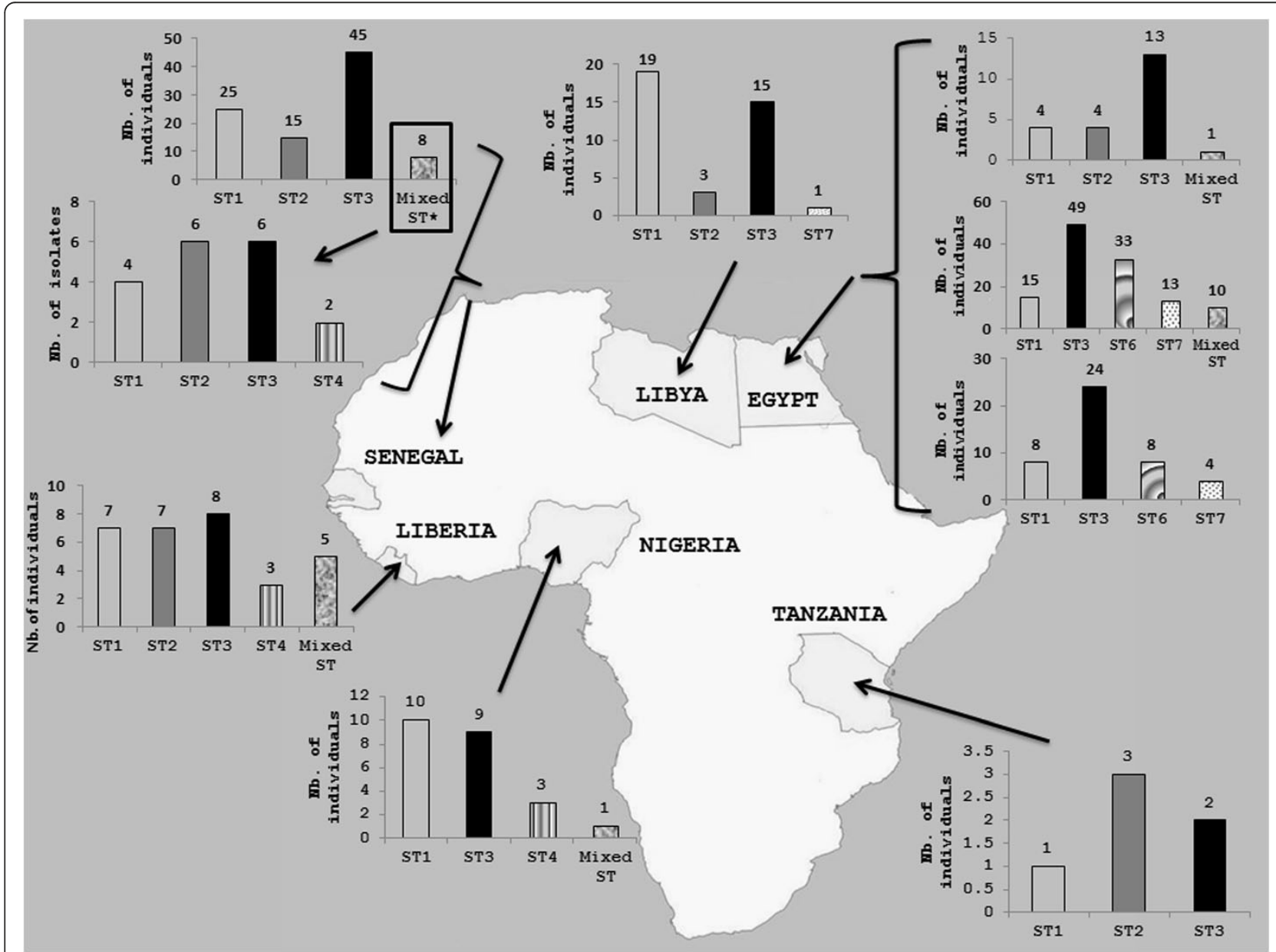

Figure 1 Distribution of Blastocystis sp. STs across African countries. In the case of Senegal, distribution of STs in mixed infections indicated by an asterisk in the histogram was detailed. 
Table 4 Distribution of Blastocystis sp. STs among symptomatic and asymptomatic children groups

\begin{tabular}{cccccc}
\hline Group & \multicolumn{3}{c}{ Blastocystis sp. STs (\%) } & Total no. \\
\cline { 2 - 5 } & ST1 & ST2 & ST3 & $\begin{array}{c}\text { Mixed } \\
\text { infections }\end{array}$ & \\
\hline Symptomatic & $13(24)$ & $7(13)$ & $28(52)$ & $6(11)$ & 54 \\
Asymptomatic & $12(31)$ & $8(20)$ & $17(44)$ & $2(5)$ & 39 \\
Total no (\%) & $\mathbf{2 5 ( 2 7 )}$ & $\mathbf{1 5 ( 1 6 )}$ & $\mathbf{4 5 ( 4 8 )}$ & $\mathbf{8 ( 9 )}$ & $\mathbf{9 3}$ \\
\hline
\end{tabular}

Australian sampling were from symptomatic individuals $[8,41]$. To complete our observations, it can also be underlined that the only two ST4 isolates found in our study were associated with symptoms but in the context of mixed ST infections (associated to ST1 or ST3). Therefore, the pathogenic potential of ST4 could not be assessed from our epidemiological data. However, a high prevalence of ST4 was recently found in Danish patients presenting with acute diarrhea [33] and Spanish symptomatic individuals [62]. Globally, no association between ST and symptom status was statistically significant from our present data.

\section{Potential risk factors for transmission of Blastocystis sp. within the Senegalese cohort}

The fecal-oral route is the main mode of transmission of Blastocystis sp. like the other common gastrointestinal parasites [1]. To reach a prevalence of $100 \%$ as in the present study, it is reasonable to argue that infection of Senegalese children by the parasite occurred through multiple transmission sources. Although we did not had the opportunity to collect and analyze environmental (mainly water samples) and animal samples, it appears clear that individuals living in small communities in a rural area with modest socio-economic status (average income of 0.5 \$ per person per day in these Senegalese villages), poor hygiene sanitation, close contact with domestic animals and livestock, and water supply directly from well and river as in our study greatly increase their risks of infection by Blastocystis sp. Indeed, recent studies have revealed a human-to-human transmission coupled with a high prevalence of the parasite in a close community of girls in Thailand [13] as well as in a rural village in Nepal [14] whose description and environment (animals reared in or next to villager dwellings and rivers as water sources of the community) are quite similar to those of Senegalese villages. In this context, zoonotic transmission of Blastocystis sp. would be another significant source of infection due to the wide range of animals potentially infected with the parasite and in close contact with humans $[2,4,55,56]$. However the impact of this mode of transmission through contact with contaminated animal fecal materials coupled with poor hand hygiene of villagers remains difficult to quantify therefore that it involves STs frequently found in humans (ST1 to ST4) as in the present study. Finally, there is a concern that water should be a main source of infection by Blastocystis sp. although large-scale waterborne outbreaks involving the parasite have not yet been documented. Ability of Blastocystis sp. cysts, the only transmissible stage of this parasite to survive for long periods of time in the environment together with their low size $[1,15]$ represent some factors that favor waterborne transmission of the parasite. In 2006, Blastocystis sp. has also been added by the World Health Organization to the list of waterborne parasites [63]. Blastocystis sp. was previously reported in drinking water, rivers, tanks, and sewage samples $[11,12,14,15,64]$. Interestingly, in these latter studies, the same STs were identified in both the water samples and the stools of consumers providing evidence for water transmission. In the Senegalese villages of the present study, river and wells represent the main water sources for drinking and cooking purposes as well as for bathing of the population. Consequently, water sources of this community potentially contaminated with human and animal feces, may largely facilitate transmission of Blastocystis sp. and should be considered as serious potential threats to consumers. Further, the analysis of fecal samples from animals living in or near the Senegalese villages and of water samples would help to confirm their epidemiological importance as sources of infection and contamination for inhabitants.

\section{Conclusions}

To our knowledge, this is the first investigation of prevalence and ST distribution of Blastocystis sp. in Senegal that, moreover, greatly expands the few data available on molecular epidemiology of this parasite in Africa. In this country, the prevalence of Blastocystis sp. in a cohort of children living in a rural area reached a peak of $100 \%$ never achieved before. Such high prevalence may reflect different exposure of individuals to animal (zoonosis) and environmental (waterborne) sources of infection together with large-scale human-to-human transmission. This study also raises questions about the real pathogenicity of Blastocystis sp. since more than half of children infected by this parasite presented various gastrointestinal symptoms and highlights the socioeconomic impact of blastocystosis in developing countries with low environmental conditions and quality of life. Finally, this epidemiological survey provides necessary information to public health authorities for urgently setting prevention (water quality assessments and primary education of dwellers regarding the modes of transmission) and control programs that should help to reduce the burden of Blastocystis sp. 


\section{Additional file}

Additional file 1: Standardized questionnaire completed for each child in the study.

\section{Abbreviations}

ST: Subtype; IBS: Irritable bowel syndrome.

\section{Competing interests}

The authors declare that they have no competing interests.

\section{Authors' contributions}

LG and MS collected stools and patient data and helped in drafting the manuscript. DES, DM, AC, PP and IW collected and analyzed PCR and sequence data and helped in drafting the manuscript. EV, GR, MH, LD, FDe and FDa conceived, designed and coordinated the study and drafted the manuscript. All authors read and approved the final manuscript.

\section{Acknowledgements}

This work was supported by grants from the Programme Orientations Stratégiques from the University of Lille 2, the Centre National de la Recherche Scientifique, the Institut Pasteur of Lille and the Biomedical Research Center EPLS (Sn). DES and MO were both supported by PhD fellowships from the Conseil National de la Recherche Scientifique and the Azm \& Saade Association from Lebanon, DM by a PhD fellowship from the Regione Autonoma della Sardegna and a post-doctoral grant from the Japanese Society for Promotion of Science (JSPS) and AC by a PhD fellowship from the Ministère Français de l'Education Nationale, de la Recherche et de la Technologie.

\section{Author details}

'Institut Pasteur de Lille, Centre d'Infection et d'Immunité de Lille (CIIL), Inserm U1019, CNRS UMR 8204, Université Lille Nord de France, 1 rue du Professeur Calmette, BP 245, 59019 Lille cedex, France. ${ }^{2}$ Centre AZM pour la Recherche en Biotechnologie et ses Applications, Laboratoire Microbiologie, Santé et Environnement, Université Libanaise, Tripoli, Lebanon. ${ }^{3}$ Biomedical Research Center Espoir pour la Santé (CRB-EPLS), Saint-Louis, Senegal. ${ }^{4}$ Microbe Division/Japan Collection of Microorganisms (JCM), RIKEN BioResource Center, Tsukuba, Japan. ${ }^{5}$ Laboratoire Microorganismes: Génome et Environnement, CNRS UMR 6023, Université Blaise Pascal de Clermont-Ferrand, Aubière, France.

Received: 22 November 2013 Accepted: 19 March 2014 Published: 25 March 2014

\section{References}

1. Tan KS: New insights on classification, identification, and clinical relevance of Blastocystis spp. Clin Microbiol Rev 2008, 21:639-665.

2. Alfellani MA, Taner-Mulla D, Jacob AS, Imeede CA, Yoshikawa H, Stensvold CR, Clark CG: Genetic diversity of Blastocystis in livestock and zoo animals. Protist 2013, 164:497-509.

3. Alfellani MA, Jacob AS, Perea NO, Krecek RC, Taner-Mulla D, Verweij J J, Levecke B, Tannich E, Clark CG, Stensvold CR: Diversity and distribution of Blastocystis sp. subtypes in non-human primates. Parasitology 2013. 140:966-971.

4. Roberts T, Stark D, Harkness J, Ellis J: Subtype distribution of Blastocystis isolates from a variety of animals from South Wales, Australia. Vet Parasitol 2013, 196:85-89.

5. Alfellani MA, Stensvold CR, Vidal-Lapiedra A, Onuoha ES, Fagbenro-Beyioku AF, Clark CG: Variable geographic distribution of Blastocystis subtypes and its potential implications. Acta Trop 2013, 126:11-18.

6. Pegelow K, Gross R, Pietrzik K, Lukito W, Richards AL, Fryauff DJ: Parasitological and nutritional situation of school children in the Sukaraja district, West Java, Indonesia. Southeast Asian J Trop Med Public Health 1997, 28:173-190.

7. Ramirez JD, Sanchez LV, Bautista DC, Corredor AF, Florez AC, Stensvold CR: Blastocystis subtypes detected in humans and animals from Colombia. Infect Genet Evol 2013. doi:10.1016/j.meegid.2013.07.020. [Epub ahead of print]
8. Roberts T, Stark D, Harkness J, Ellis J: Subtype distribution of Blastocystis isolates identified in a Sydney population and pathogenic potential of Blastocystis. Eur J Clin Microbiol Infect Dis 2013, 32:335-343.

9. Bart A, Wentink-Bonnema EMS, Gilis H, Verhaar N, Wassenaar CJA, van Vug M, Goorhuis A, van Gool T: Diagnosis and subtype analysis of Blastocystis sp. in 442 patients in a hospital setting in the Netherlands. BMC Infect Dis 2013, 13:389.

10. Li LH, Zhou XN, Du ZW, Wang XZ, Wang LB, Jiang JY, Yoshikawa H, Steinmann P, Utzinger J, Wu Z, Chen JX, Chen SH, Zhang L: Molecular epidemiology of human Blastocystis in a village in Yunnan province, China. Parasitol Int 2007, 56:281-286.

11. Leelayoova S, Siripattanapipong S, Thathaisong U, Naaglor T, Taamasri $P$ Piyaraj P, Mungthin M: Drinking water: a possible source of Blastocystis spp. subtype 1 infection in schoolchildren of a rural community in central Thailand. Am J Trop Med Hyg 2008, 79:401-406.

12. Eroglu F, Koltas IS: Evaluation of the transmission mode of $B$. hominis by using PCR method. Parasitol Res 2010, 107:841-845.

13. Thathaisong U, Siripattanapipong S, Mungthin M, Pipatsatitpong D, Tan-ariya P, Naaglor T, Leelayoova S: Identification of Blastocystis subtype 1 variants in the home for girls, Bangkok, Thailand. Am J Trop Med Hyg 2013, 88:352-358.

14. Lee LL, Chye T, Karmacharya BM, Govind SK: Blastocystis sp.: waterborne zoonotic organism, a possibility? Parasit Vectors 2012, 5:130.

15. Suresh K, Smith H, Tan T: Viable Blastocystis cysts in Scottish and Malaysian sewage samples. Appl Environ Microbiol 2005, 71:5619-5620.

16. Yoshikawa H, Wu Z, Pandey K, Pandey BD, Sherchand JB, Yanagi T, Kanbara $\mathrm{H}$ : Molecular characterization of Blastocystis isolates from children and rhesus monkeys in Kathmandu, Nepal. Vet Parasitol 2009, 160:295-300.

17. Parkar U, Traub RJ, Vitali S, Elliot A, Levecke B, Robertson I, Geurden T, Steele J, Drake B, Thompson RC: Molecular characterization of Blastocystis isolates from zoo animals and their animal-keepers. Vet Parasitol 2010, 169:8-17

18. Noël C, Dufernez F, Gerbod D, Edgcomb VP, Delgado-Viscogliosi P, Ho LC, Singh M, Wintjens R, Sogin ML, Capron M, Pierce R, Zenner L, Viscogliosi E: Molecular phylogenies of Blastocystis isolates from different hosts: implications for genetic diversity, identification of species, and zoonosis. J Clin Microbiol 2005, 43:348-355.

19. Stensvold CR, Suresh GK, Tan KS, Thompson RC, Traub RJ, Viscogliosi E, Yoshikawa H, Clark CG: Terminology for Blastocystis subtypes-a consensus. Trends Parasitol 2007, 23:93-96.

20. Poirier P, Wawrzyniak I, Albert A, El AH, Delbac F, Livrelli V: Development and evaluation of a real-time PCR assay for detection and quantification of Blastocystis parasites in human stool samples: prospective study of patients with hematological malignancies. J Clin Microbiol 2011, 49:975-983.

21. Hussein EM, Hussein AM, Eida MM, Atwa MM: Pathophysiological variability of different genotypes of human Blastocystis hominis egyptian isolates in experimentally infected rats. Parasitol Res 2008, 102:853-860.

22. Tan KSW, Mirza H, Teo JDW, Wu B, MacAry PA: Current views on the clinical relevance of Blastocystis spp. Curr Infect Dis Rep 2010, 12:28-35.

23. Denoeud F, Roussel M, Noël B, Wawrzyniak I, Da Silva C, Diogon M, Viscogliosi E, Brochier-Armamet C, Couloux A, Poulain J, Segurans B, Anthouard V, Texier C, Blot N, Poirier P, Ng GC, Tan KSW, Antiguenave F, Jaillon O, Aury J, Delbac F, Wincker P, Vivarès CP, El Alaoui H: Genome sequence of the stramenopile Blastocystis, a human anaerobic parasite. Genome Biol 2011, 12:R29.

24. Clark CG, van der Giezen M, Alfellani MA, Stensvold CR: Recent developments in Blastocystis research. Adv Parasitol 2013, 82:1-32.

25. Poirier P, Wawrzyniak I, Vivares CP, Delbac F, El AH: New insights into Blastocystis spp.: a potential link with irritable bowel syndrome. PLoS Pathog 2012, 8:e1002545.

26. Mirza H, Wu Z, Teo JDW, Tan KSW: Statin pleiotropy prevents rho kinasemediated intestinal epithelial barrier compromise induced by Blastocystis cysteine proteases. Cell Microbiol 2012, 14:1474-1484.

27. Wawrzyniak I, Texier C, Poirier P, Viscogliosi E, Tan KS, Delbac F, El Alaoui H: Characterization of two cysteine proteases secreted by Blastocystis ST7, a human intestinal parasite. Parasitol Int 2012, 61:437-442.

28. Boorom KF, Smith H, Nimri L, Viscogliosi E, Spanakos G, Parkar U, Li LH, Zhou XN, Ok UZ, Leelayoova S, Jones MS: Oh my aching gut: irritable bowel syndrome, Blastocystis, and asymptomatic infection. Parasit Vectors 2008, 1:40. 
29. Vogelberg C, Stensvold CR, Monecke S, Ditzen A, Stopsack K, Heinrich-Grafe $U$, Pohlmann C: Blastocystis sp. subtype 2 detection during recurrence of gastrointestinal and urticarial symptoms. Parasitol Int 2010, 59:469-471.

30. Verma R, Delfanian K: Blastocystis hominis associated acute urticaria. Am J Med Sci 2013, 346:80-81

31. Tan TC, Ong SC, Suresh KG: Genetic variability of Blastocystis sp. isolates obtained from cancer and HIV/AIDS patients. Parasitol Res 2009, 105:1283-1286.

32. Batista MW, Pierrotti LC, Abdala E, Clemente WT, Girao ES, Rosa DRT, lanhez LE, Bonazzi PR, Lima AS, Fernandes PFCBC, Padua-Neto MV, Bacchella T, Oliveira APP, Viana CFG, Ferreira MS, Shikanai-Yasuda MA: Endemic and opportunistic infections in Brazilian solid organ transplant recipients. Trop Med Int Health 2011, 16:1134-1142.

33. Stensvold CR, Christiansen DB, Olsen KEP, Nielsen HV: Blastocystis sp. subtype 4 is common in Danish Blastocystis-positive patients presenting with acute diarrhea. Am J Trop Med Hyg 2011, 84:883-885.

34. Forsell J, Granlund M, Stensvold CR, Clark CG, Evengard B: Subtype analysis of Blastocystis isolates in Swedish patients. Eur J Clin Microbiol Infect Dis 2012, 31:1689-1696.

35. Moosavi A, Haghighi A, Nazemalhosseini Mojarad E, Zayeri F, Alebouyeh M, Khazan H, Kazemi B, Zali MR: Genetic variability of Blastocystis sp. isolated from symptomatic and asymptomatic individuals in Iran. Parasitol Res 2012, 111:2311-2315.

36. El Safadi D, Meloni D, Poirier P, Osman M, Cian A, Gaayeb L, Wawrzyniak I, Delbac F, El Alaoui H, Delhaes L, Dei-Cas E, Mallat H, Dabboussi F, Hamze M, Viscogliosi E: Molecular epidemiology of Blastocystis in Lebanon and correlation between subtype 1 and gastrointestinal symptoms. Am J Trop Med Hyg 2013, 88:1203-1206.

37. Souppart L, Moussa H, Cian A, Sanciu G, Poirier P, El Alaoui H, Delbac F, Boorom K, Delhaes L, Dei-Cas E, Viscogliosi E: Subtype analysis of Blastocystis isolates from symptomatic patients in Egypt. Parasitol Res 2010 106:505-511.

38. Fouad SA, Basyoni MM, Fahmy RA, Kobaisi MH: The pathogenic role of different Blastocystis hominis genotypes isolated from patients with irritable bowel syndrome. Arab J Gastroenterol 2011, 12:194-200.

39. Petrasova J, Uzlikova M, Kostka M, Petrzelkova KJ, Huffman MA, Modry D: Diversity and host specificity of Blastocystis in syntopic primates on Rubondo Island, Tanzania. Int J Parasitol 2011, 41:1113-1120.

40. Scicluna SM, Tawari B, Clark CG: DNA barcoding of Blastocystis. Protist 2006 157:77-85.

41. Souppart L, Sanciu G, Cian A, Wawrzyniak I, Delbac F, Capron M, Dei-Cas E, Boorom K, Delhaes L, Viscogliosi E: Molecular epidemiology of human Blastocystis isolates in France. Parasitol Res 2009, 105:413-421.

42. Stensvold CR: Blastocystis: genetic diversity and molecular methods for diagnosis and epidemiology. Trop Parasitol 2013, 3:26-34

43. Stensvold CR: Comparison of sequencing (barcode region) and sequence-tagged-site PCR for Blastocystis subtyping. J Clin Microbiol 2013, 51:190-194.

44. Meloni D, Poirier P, Mantini C, Noël C, Gantois N, Wawrzyniak I, Delbac F, Chabé M, Delhaes L, Dei-Cas E, Fiori PL, El Alaoui H, Viscogliosi E: Mixed human intra- and inter-subtype infections with the parasite Blastocystis sp. Parasitol Int 2012, 61:719-722.

45. Velasco J, Gonzalez F, Diaz T, Pena-Guillen J, Araque M: Profiles of enteropathogens in asymptomatic children from indigenous communities of Merida, Venezuela. J Infect Dev Ctries 2011, 5:276-285.

46. Machicado JD, Marcos LA, Tello R, Canales M, Terashima A, Gotuzzo E: Diagnosis of soil-transmitted helminthiasis in an Amazonic community in Peru using multiple diagnostic techniques. Trans $R$ Soc Trop Med Hyg 2012, 106:333-339

47. Saksirisampant W, Nuchprayoon S, Wiwanitkit V, Yenthakam S, Ampavasiri A: Intestinal parasitic infestations among children in an orphanage in Patum Thani province. J Med Assoc Thai 2003, 86:S263-S270.

48. Gualdieri L, Rinaldi L, Petrullo L, Morgoglione ME, Maurelli MP, Musella V, Piemonte M, Caravano L, Coppola MG, Cringoli G: Intestinal parasites in immigrants in the city of Naples (southern Italy). Acta Trop 2011, 117:196-201.

49. Alfellani MA, Khan AH, Al-Gazaoui RM, Zaid MK, Al-Ferjani MA: Prevalence and clinical features of Blastocystis hominis infection among patients in Sebha, Libya. Sultan Qaboos Univ Med J 2007, 7:35-40.

50. Faye O, Ndir O, Dieng T, Gaye O, Bah IB, Dieng Y, Faye I, Diallo S: Cryptosporidiosis among intestinal parasitosis in Senegalese pediatric hospital patients. Dakar Med 1993, 38:129-132.
51. Gaye O, Ndir O, Kane A, Belmachi R, Ndiaye M, Diouf M, Diedhiou M, Diallo S: Intestinal parasitic diseases and tinea of the scalp in Dakar school population: influence of environmental factors on the infestation level. Dakar Med 1994, 39:57-61.

52. Salem G, van de Velden L, Laloé F, Maire B, Ponton A, Traissac P, Prost A: Intestinal parasitic diseases and environment in Sahelo-Sudanese towns: the case of Pikine (Senegal). Rev Epidemiol Sante Publique 1994, 42:322-333

53. Ndir I, Gaye A, Sy M, Gaye O, Ndir O: Prevalence of intestinal parasites at the King Baudouin health center of Guediawaye (Senegal). Dakar Med 2002, 47:168-171.

54. Stensvold CR, Alfellani M, Clark CG: Levels of genetic diversity vary dramatically between Blastocystis subtypes. Infect Genet Evol 2012, 12:263-273

55. Tian CT, Tan PC, Sharma R, Sugnaseelan S, Suresh KG: Genetic diversity of caprine Blastocystis from Peninsular Malaysia. Parasitol Res 2013, 112:85-89.

56. Stensvold CR, Alfellani MA, Norskov-Lauritsen S, Prip K, Victory EL, Maddox C, Nielsen HV, Clark CG: Subtype distribution of Blastocystis isolates from synanthropic and zoo animals and identification of a new subtype. Int Parasitol 2009, 39:473-479.

57. Dogruman-Al F, Dagci H, Yoshikawa H, Kurt Ö, Demirel M: A possible link between subtype 2 and asymptomatic infections of Blastocystis hominis Parasitol Res 2008, 103:685-689.

58. Özyurt M, Kurt Ö, Molbak K, Nielsen HV, Haznedaroglu T, Stensvold CR: Molecular epidemiology of Blastocystis infection in Turkey. Parasitol Int 2008, 57:300-306.

59. Li J, Deng T, Li X, Cao G, Li X, Yan Y: A rat model to study Blastocystis subtype 1 infections. Parasitol Res 2013, 112:3537-3541.

60. Eroglu F, Gene A, Elgun K, Koltas IS: Identification of Blastocystis hominis isolates from asymptomatic and symptomatic patients by PCR. Parasitol Res 2009, 105:1589-1592.

61. Yakoob J, Jafri W, Beg MA, Abbas Z, Naz S, Islam M, Khan R: Irritable bowel syndrome: is it associated with genotypes of Blastocystis hominis. Parasitol Res 2010, 106:1033-1038.

62. Dominguez-Marquez MV, Guna R, Munoz C, Gomez-Munoz MT, Borras R: High prevalence of subtype 4 among isolates of Blastocystis hominis from symptomatic patients of a health district of Valencia (Spain). Parasitol Res 2009, 105:949-955.

63. World Health Organization: Microbial fact sheets. In World Health Organization Guidelines for Drinking Water quality (WHOGDWQ). 4th edition. Malta: Gutenberg; 2011:271-273.

64. Leelayoova S, Rangsin R, Taamasri P, Naaglor T, Thathaisong U, Mungthin M: Evidence of waterborne transmission of Blastocystis hominis. Am J Trop Med Hyg 2004, 70:658-662.

doi:10.1186/1471-2334-14-164

Cite this article as: El Safadi et al.: Children of Senegal River Basin show the highest prevalence of Blastocystis sp. ever observed worldwide. BMC Infectious Diseases 2014 14:164.

\section{Submit your next manuscript to BioMed Central and take full advantage of:}

- Convenient online submission

- Thorough peer review

- No space constraints or color figure charges

- Immediate publication on acceptance

- Inclusion in PubMed, CAS, Scopus and Google Scholar

- Research which is freely available for redistribution 